\section{P37 A MODEL FOR REDUCING PAEDIATRIC PRESCRIBING ERRORS IN SECONDARY CARE}

Kate Morgan*. Royal Gwent Hospital

10.1136/archdischild-2020-NPPG.46

Background A prescribing error is a preventable error that may lead to inappropriate medication use and patient harm (1). Prescribing errors are particularly important in paediatrics where dose calculations are complicated and small errors can result in significant morbidity and mortality. ${ }^{1}$ In 2017 pharmacy data showed that paediatric prescribing errors were an issue at our Hospital regarding the severity and high numbers of errors, especially for antibiotics and analgesia.

Objectives To achieve a zero prescribing error rate for paediatric within the hospital.

Method

1. Form the Paediatric Medication Errors Prevention (PMEP) group consisting of the Paediatric Consultant, Paediatric Pharmacist, Children's Assessment Unit Sister and Practice Education Senior Nurse.

2. Paediatric Pharmacist to record and feedback all paediatric prescribing errors weekly at Doctors' handover.

3. Paediatric Pharmacist/Nurses to DATIX report all significant medication prescribing errors

4. Paediatric Pharmacist to produce and communicate monthly pharmacy prescribing newsletter.

5. Paediatric Pharmacist to produce quick reference charts for the drugs with the most common prescribing errors e.g.

antibiotics and analgesia

6. Paediatric Doctors to request a second check from another Doctor or Ward Sister when prescribing any medication on the drug chart of take home prescription.

7. Paediatric Pharmacist to target Doctors' induction to improve prescribing and implement a prescribing test.

8. Doctors to complete reflections for errors with their educationsal supervisors.

This study did not require ethics approval.

Results Following implementation of the above strategies, there was a $33 \%$ reduction in the number of prescribing errors recorded by the Paediatric Pharmacist daily intervention $\log$ from 2017/2018 to 2018/2019. There were 163 prescribing errors for 2017/2018 compared to 110 for 2018/ 2019.

Conclusion The formation of the PMEP group and implementation of strategies to reduce paediatric prescribing errors has positively impacted on reducing the error rate at the hospital. It has also raised awareness of the necessity to report all errors and actively find ways to prevent these from re-occurring. Further work is required to reduce these errors to zero including targeting non paediatric teams prescribing on paediatrics and implementing Pharmacists prescribing on consultant ward rounds. Future work would also include replicating this model in other specialities e.g. neonatal intensive care to achieve the same success rate in reducing medication errors.

\section{REFERENCE}

1. Davis T. Paediatric prescribing errors. Arch Dis Child. 2011;96:489-91. Accessed via http://adc.bmj.com on 2/4/19.

\section{P38 \\ AN AUDIT OF ANTIBIOTIC USE IN THE TREATMENT OF NEONATAL NECROTISING ENTEROCOLITIS (NEC): WHAT DO WE USE AND HOW LONG FOR?}

Stephen Morris, Kimberly Mak* ${ }^{*}$ Rebecca Lisseter. Leeds Teaching Hospitals NHS Trust

10.1136/archdischild-2020-NPPG.47

Introduction Necrotising enterocolitis (NEC) is a serious condition in premature infants involving inflammation, and potentially necrosis, of the bowel. Several contributory factors have been identified; including prematurity, infection and reduced gut perfusion. ${ }^{1}$ It has previously been reported that there is widespread variation in the treatment of NEC ${ }^{2}$, including the use of antibiotics. ${ }^{3}$ This is due to a lack of evidence evaluating which treatment option is most effective.

Optimising the use of antibiotics for treating NEC is an opportunity to support the wider, global initiative of antimicrobial stewardship. ${ }^{4}$ Ensuring we use only the necessary course of antibiotics will reduce the spread of resistance and lower risks of potential adverse effects. Our local NEC treatment guideline states that the first line antibiotic regimen should be amoxicillin, gentamicin and metronidazole for up to 10 days treatment.

Aim The aim of our audit was to identify the type of antibiotics and the duration of therapy used to treat NEC within a single UK tertiary children's hospital providing neonatal intensive care and neonatal surgery.

Methods A retrospective audit was conducted over a five month period between August and December 2018. The neonatal database $\left(\right.$ Badger $^{\circledR}$ ) was used to identify patients with a confirmed diagnosed of NEC. Our electronic prescribing software $\left(\right.$ MedChart ${ }^{\circledR}$ ) along with patient medical notes was then used to determine the antibiotics used and the prescribed length of treatment. A re-audit was also conducted over a 5 month period between January and May 2019.

Results We identified seven patients with confirmed diagnosis of NEC. The median gestational age was $30+6$ weeks (range $29+4$ to $36+1$ weeks) and the median birth weight was 1530 grams (range 780 to 2100 grams). Four patients required surgery that involved laparotomies and bowel resections. No patients had allergies to penicillins.

The most frequently prescribed antibiotic regimen was ceftazidime, vancomycin and metronidazole $(n=4)$. Other regimens included meropenem and vancomycin $(n=2)$, and amoxicillin, gentamicin and metronidazole $(\mathrm{n}=1)$. The median course length was 10 days (range 2 to 16 days). These results were presented to a neonatal surgery quality improvement meeting in January 2019.

During the re-audit period, five infants were identified. The median gestation was $28+3$ weeks $(26+1$ to $37+0$ weeks) and median birth weight of 1310 grams (range 6202090 grams). 3 patients had laparotomies and none had allergies to penicillins. In this cohort, the most popular regimen was amoxicillin, gentamicin and metronidazole $(n=4)$, followed by ceftazidime, vancomycin, and metronidazole $(\mathrm{n}=1)$. The median course length was 11 days (range 5 to 12 days).

Conclusion Our initial audit confirmed that considerable variation still exists in the antibiotic regimen used and the duration of treatment. We also found that patients were 